\title{
Study on CRE arrival distributions with PAMELA experiment
}

\section{B. Panico*, D. Campana, G. Osteria}

INFN, Sezione di Napoli, I-80126 Naples, Italy

E-mail: beatrice.panico@na.infn.it

G. C. Barbarino, V. Scotti

University of Naples 'Federico II', Department of Physics, I-80126 Naples, Italy

INFN, Sezione di Napoli, I-80126 Naples, Italy

O. Adriani, M. Bongi, P. Spillantini, N. Mori

University of Florence, Department of Physics and Astronomy, I-50019 Sesto

Fiorentino, Florence, Italy

INFN, Sezione di Firenze, I-50019 Sesto Fiorentino, Florence, Italy

P. Papini, E. Vannuccini, S. Bottai

INFN, Sezione di Firenze, I-50019 Sesto Fiorentino, Florence, Italy

G. A. Bazilevskaya, A. N. Kvashnin, Y. I. Stozhkov

Lebedev Physical Institute, RU-119991, Moscow, Russia

\section{R. Bellotti, A. Monaco}

University of Bari, Department of Physics, I-70126 Bari, Italy

INFN, Sezione di Bari, I-70126 Bari, Italy

\section{A. Bruno}

University of Bari, Department of Physics, I-70126 Bari, Italy

\section{F. Cafagna}

INFN, Sezione di Bari, I-70126 Bari, Italy

M. Boezio, V. Bonvicini, E. Mocchiutti, A. Vacchi, G. Zampa, N. Zampa

INFN, Sezione di Trieste, I-34149 Trieste, Italy

\section{E. A. Bogomolov, S. Y. Krutkov, G. I. Vasilyev}

Ioffe Physical Technical Institute, RU-194021 St. Petersburg, Russia

\section{P. Carlson,M. Pearce}

KTH, Department of Physics, and the Oskar Klein Centre for Cosmoparticle Physics, AlbaNova University Centre, SE-10691 Stockholm, Sweden 


\section{Casolino}

INFN, Sezione di Roma Tor Vergata, I-00133 Rome, Italy

RIKEN, Advanced Science Institute, Wako-shi, Saitama, Japan

\section{G. Castellini}

IFAC, I-50019 Sesto Fiorentino, Florence, Italy

\section{De Donato, C. De Santis, L. Marcelli, M. Mergé, F. Palma, P. Picozza, R. Sparvoli} University of Rome Tor Vergata, Department of Physics, I-00133 Rome, Italy INFN, Sezione di Roma Tor Vergata, I-00133 Rome, Italy

\section{N. De Simone}

INFN, Sezione di Roma Tor Vergata, I-00133 Rome, Italy

\section{Di Felice}

INFN, Sezione di Roma Tor Vergata, I-00133 Rome, Italy

Agenzia Spaziale Italiana (ASI) Science Data Center, Via del Politecnico snc I-00133 Rome, Italy

\section{Formato, R. Munini}

University of Trieste, Department of Physics, I-34147 Trieste, Italy

INFN, Sezione di Trieste, I-34149 Trieste, Italy

\section{A. M. Galper,A. V. Karelin,S. V. Koldashov,S. Koldobskiy,Y. T. Yurkin,V. Malakhov,A.} Leonov,A. G. Mayorov,V. V. Mikhailov,S. A. Voronov

National Research Nuclear University MEPhI, RU-115409 Moscow

\section{U. Giaccari}

INFN, Sezione di Napoli, I-80126 Naples, Italy

Now at Universidade Federal do Rio de Janeiro, Instituto de Fisica, Rio de Janeiro, RJ, Brazil

\section{Martucci}

University of Rome Tor Vergata, Department of Physics, I-00133 Rome, Italy

INFN, Laboratori Nazionali di Frascati, Via Enrico Fermi 40, I-00044 Frascati, Italy

\section{W. Menn, M. Simon}

Universitat Siegen, Department of Physics, D-57068 Siegen, Germany

\section{Ricci}

INFN, Laboratori Nazionali di Frascati, Via Enrico Fermi 40, I-00044 Frascati, Italy

\section{S. B. Ricciarini}

INFN, Sezione di Firenze, I-50019 Sesto Fiorentino, Florence, Italy

IFAC, I-50019 Sesto Fiorentino, Florence, Italy 
Abstract: From 2009, several experiments, like PAMELA, FERMI and AMS, have shown a rise in the fraction of positrons versus electrons+positrons. One of the most probable explanation is due to the presence of nearby sources, like SNRs or pulsars. PAMELA (Payload for Antimatter Matter Exploration and Light-nuclei Astrophysics) is a ballooon-borne experiment and is collecting data since 15 June 2006. Its quasi-polar orbit permits to perform a survey in each direction of the sky. The study of the arrival distribution of cosmic ray electrons and positrons from different regions allows the exploration of different origins for the excess.

The 34th International Cosmic Ray Conference, 30 July - 6 August, 2015

The Hague, The Netherlands

${ }^{*}$ Speaker. 


\section{Introduction}

Cosmic rays are diffused inside the Galaxy for a very long time under the effect of magnetic fields and their arrival direction distribution is highly randomized [1]. Since 80's has been clearly observed an energy-dependent anisotropy of galactic cosmic rays, both in the northern $[2,3]$ and in the southern hemisphere [4]. Anisotropy studies can lead to a better understanding of cosmic ray propagation into the local interstellar medium. Furthermore, contrary to hadrons, cosmic-ray electrons and positrons (CREs) lose rapidly their energy, due to synchrotron radiation emission and inverse Compton collisions with low-energy photons of the interstellar radiation field. These losses limit distances over which CREs can remain highly energetic. Recently PAMELA [5], Fermi-LAT [6] and AMS-02 [7] observed a rise in the positron fraction above $10 \mathrm{GeV}$. Since this rise is not compatible with the cosmic-ray secondary production, additional sources of primary CREs have been proposed. Such sources may be either of astrophysical origin, like supernovae [8] or pulsars [9], or due to dark matter annihilation [10]. A way to distinguish between different origins is represented by the detection of an excess/deficit in the arrival direction of CREs. Astrophysical sources need to be relatively close at these energies, thus implying an anisotropy in the local CR positron flux. On the contrary, models using dark matter to explain the cosmic-ray excess forecast significant anisotropies in the arrival direction together with the production of a bright gamma-ray emission, that would be easily detectable by space experiments [11]. A method for the detection of positron anisotropy with PAMELA data has been described in [12]. In this paper the analysis on a sample of electrons selected as in [5] is reported, taking into account also the heliosperic and geomagnetic effects.

\section{The PAMELA experiment}

PAMELA (Payload for AntiMatter Matter Exploration and Light-nuclei Astrophysics) is a space-based cosmic-ray detector hosted on the Russian satellite Resurs DK1. It was launched from the Bajkonour site in Kazakhistan on June 2006 and is still taking data. Its orbit is quasi-polar and elliptical with an inclination of $\sim 70^{\circ}$, and goes from 350 to $600 \mathrm{~km}$ of altitude. PAMELA apparatus is schematically showed in Fig. 1 and is accurately described in [13]. It is equipped with the following different detectors.

- A time of flight system (ToF), which is composed by 6 layers of plastic scintillators. It provides the determination of particle velocities, allows the identification of albedo particles and the proton-electron separation below $1 \mathrm{GeV} / \mathrm{c}$.

- A spectrometer, which is composed by a permanent magnet equipped with six double-sided micro-strip silicon sensors. It defines the determination of the sign of the charge and the rigidity of particles up to $1 \mathrm{TeV} / \mathrm{c}$. By measuring the ionization losses into the silicon planes, also the particle charge is obtained.

- An anticoincidence system for the rejection of secondary particles produced in the interaction with the mechanical structure of the payload. 


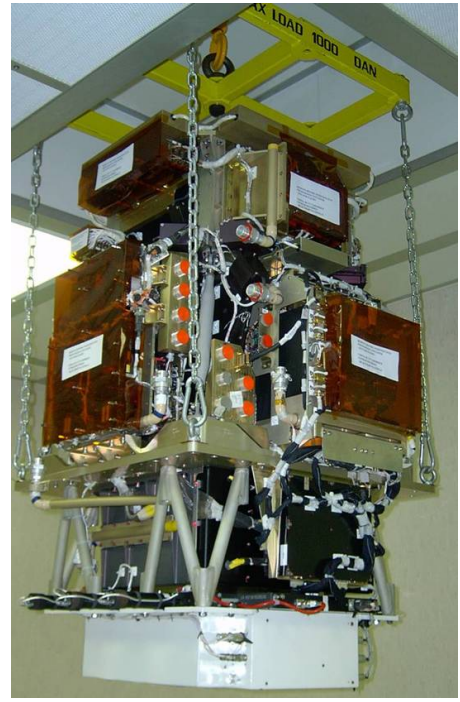

(a)

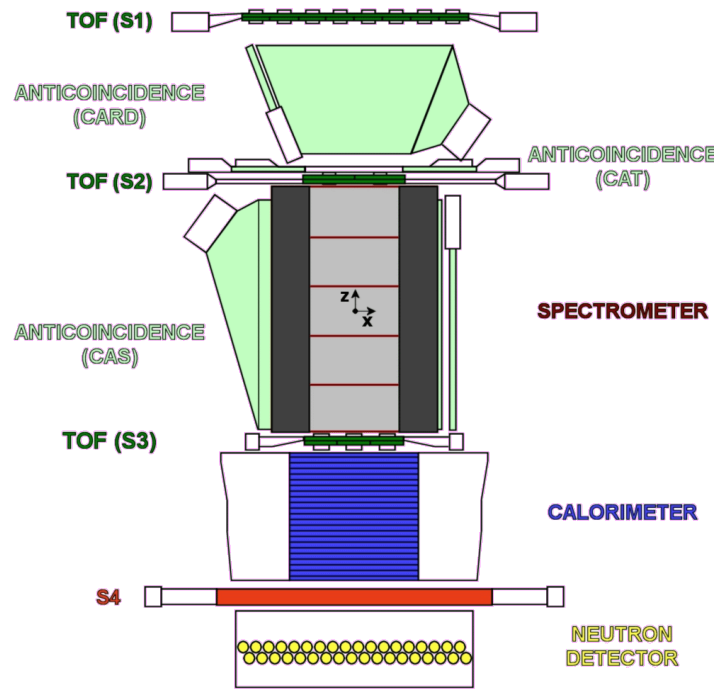

(b)

Figure 1: (a) The PAMELA experiment. (b) A schematic view of the apparatus.

- An electromagnetic calorimeter, which is made up of 44 silicon planes and 22 plates of tungsten. It provides a direct measurement of the energy for electrons and positrons and, through the analysis of shower topology, allows the discrimination between hadrons and leptons.

- A neutron detector which improves the particle discrimination.

\section{The analysis of an electron sample}

The analysis has been performed on a sample of $\sim 2 \cdot 10^{4}$ selected electrons in the rigidity range from 10 to $200 \mathrm{GV}$. The selection procedure and the method are accurately described in [14]. For each event the particle arrival direction is reconstructed using the trajectory inside the instrument and the satellite position on the orbit. The accuracy is about 2 degrees over the whole energy range. All sky maps are produced with Healpix software [15], which pixelises the entire sphere with same area pixels. The chosen angular pixel extension is $\sim 7^{\circ}$ and the Galactic reference system is used. The map of the events is shown in the plot (a) of Fig. 2.

The map of arrival distributions needs to be compared with a reference map which represented the cosmic-ray background generated by an isotropic flux. The background map corresponds to the 'null' hypothesis and is obtained with a MonteCarlo approach. About $10^{6}$ electrons in the rigidity range 10-200 GeV have been randomly produced on a generation surface placed above the aluminium container of the instrument. An isotropic CRE intensity has been simulated by randomly choosing the generation point and the incident angles, according to an uniform distribution on the surface and in a $2 \pi$ solid angle. The same selection criteria used for data have been applied to the simulation sample. For each selected event, orbital information have been retrieved from data 


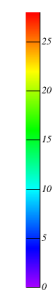

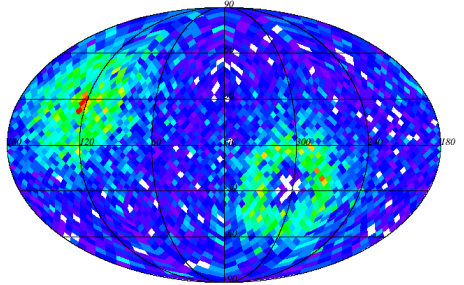

(a)

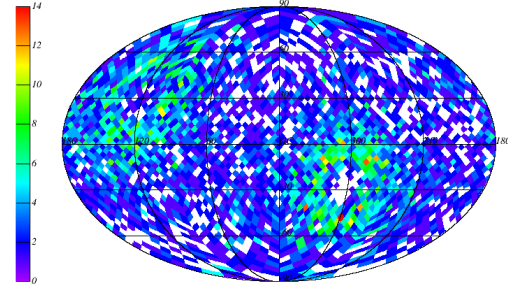

(b)

Figure 2: (a) Event map for electrons with rigidity $10 \mathrm{GV}<\mathrm{R}<200 \mathrm{GV}$. (b) Map of simulated electrons with rigidity $10 \mathrm{GV}<\mathrm{R}<200 \mathrm{GV}$. The color scale represents the occupancy of each pixel.

itself. The background map is reported in plot (b) of Fig.2.

To increase sensitivity for the detection of the anisotropy, the bin width needs to be comparable to the angular size of the signal. Since the size of the anisotropy signal is unknow, the analysis has been done on different angular scales. Signal and background maps have been integrated on four different radii, $10^{\circ}, 30^{\circ}, 60^{\circ}, 90^{\circ}$. For each integration radius, a statistical significance test has been performed to compare maps.

The significance is evaluated as indicated by $\mathrm{Li}$ and $\mathrm{Ma}$ in [16], comparing signal and background maps. Results are reported in Fig. 3, where the color scale represents the calculated significance for each pixel.

\section{The backtracing procedure}

The effects of the Earth's magnetic field could smear a weak anisotropy, indeed particles were backtraced up to 25 Earth radii on the basis of numerical integration methods [17]. The main magnetic field is described using the IGRF-11 [18] model; the TS05 [19] model is used for the external geomagnetic field sources in the inner magnetosphere. This procedure has been applied to previously selected electrons; the map corresponding to backtraced events up to 25 Earth radii is reported in Fig.4a. The backtracing procedure is also applied to simulated data; the result is shown in the plot (b) of Fig. 4.

A statistical analysis is applied to search for an anisotropy signal at the different angular scales previously indicated. The comparison with Li \& Ma tecnique between the signal and background map, after the application of the backtracing procedure, has been done. Results have been reported in Fig. 5 for the following integration radii: $10^{\circ}, 30^{\circ}, 60^{\circ}, 90^{\circ}$.

\section{Conclusions}

The favoured explanation for PAMELA results on positron fraction is represented by the existence of additional cosmic-ray sources. A measurement of an anisotropy signal in the arrival direction of CREs might help to distinguish between dark matter and astrophysical origin of predicted sources. In this paper the analysis on the arrival distribution of PAMELA data has been 

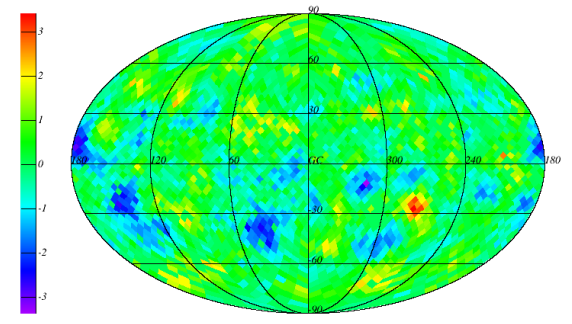

(a)

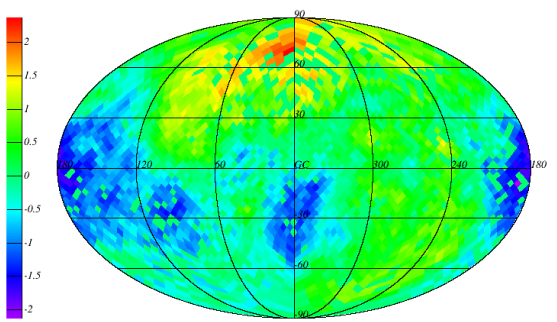

(c)

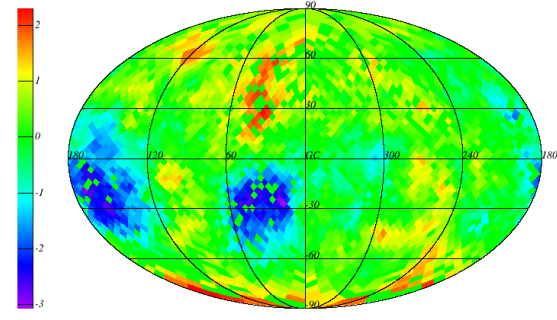

(b)

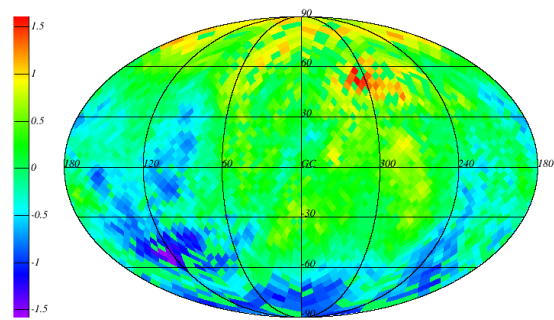

(d)

Figure 3: Significance maps obtained applying the tecnique described in [16] on real and simulated electron maps. Results obtained on four different angular scale are reported: $10^{\circ}$ (a), $30^{\circ}$ (b), $60^{\circ}$ (c), $90^{\circ}(\mathrm{d})$.

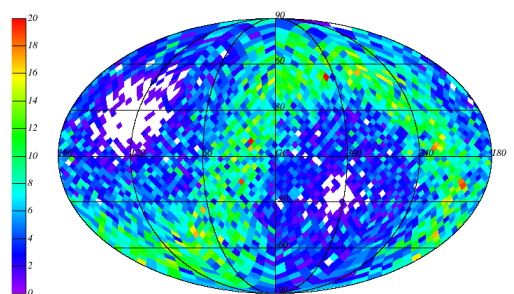

(a)

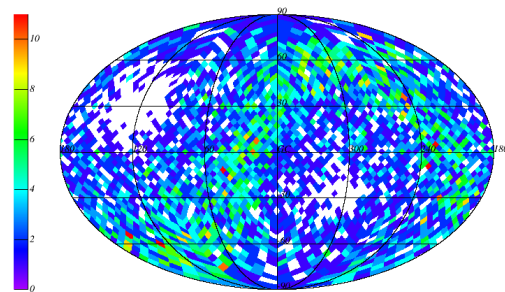

(b)

Figure 4: (a) Backtrace procedure is applied on selected electron data set. (b) The backtracing procedure is applied to events obtained with Monte Carlo simulation.

carried out. Results are consistent with isotropy at all angular scales considered. To take into account the effect of geomagnetic field particles have been backtraced up to 25 Earth's radii. The statistical analysis applied doesn't highlight significative deviation from the isotropy.

\section{References}

[1] Longair, M.S.: 1992, in High energy astrophysics, Cambridge University Press

[2] Nagashima, K., Fujimoto, K., \& Jacklyn, R.M., 1998, J. of Geophys. Res, 17429 

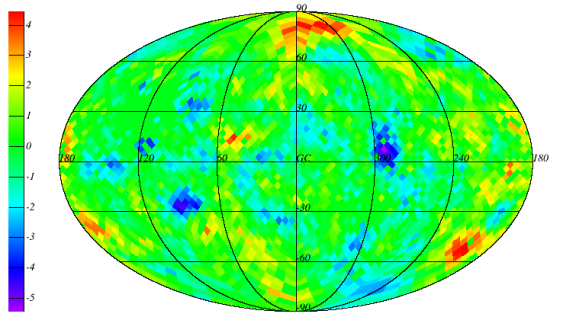

(a)

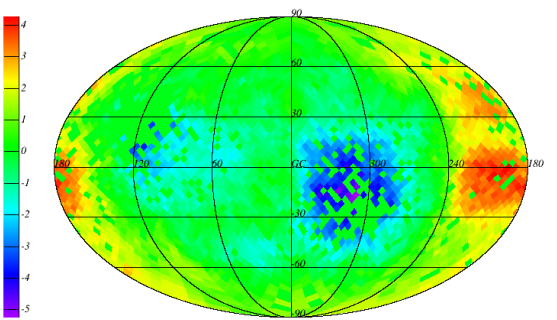

(c)

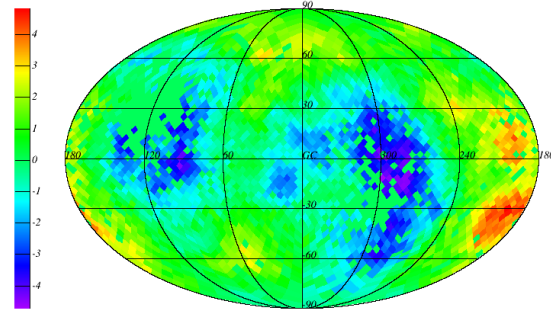

(b)

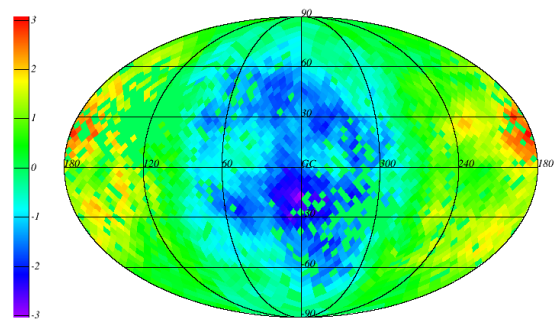

(d)

Figure 5: Maps of the significance calculated applying the Li \& Ma tecnique for the comparison of backtraced maps at four different angular scale: $10^{\circ}$ (a), $30^{\circ}(\mathrm{b}), 60^{\circ}$ (c), $90^{\circ}$ (d).

[3] Aglietta, M., Alekseenko, V.V., \& Alessandro, B. et al., 2009, ApJL, 692, L130

[4] Abbasi,R., Abdou, Y., \& Abu-Zayyad, T. et al., 2010, ApJL, 718, L194.

[5] Adriani, O., Barbarino, G. C., \& Bazilevskaya, G. A. et al., 2009, Nature, 458, 607

[6] Ackermann, M., Ajello, M., \& Allafort, A. et al., 2012, PhRvL, 108, 011103

[7] Accardo, L., Aguilar, M. \& Aisa, D. et al. 2014, PhRvL, 113, 121101

[8] Blasi, P. 2009, PRL, 103, 051104

[9] Hooper, D., Blasi, P., \& Serpico, D. 2009, JCAP, 0901, 025

[10] Grasso, D. et al. 2009, APh., 32, 140

[11] Brun, P., Delahaye, T., Diemand, J., Profumo, S., \& Salati, P. 2009, PhRvD, 80, 035023

[12] Panico, B. Adriani, O., \& Barbarino, G. C. et al., 2014, NuPhS, 256, 173

[13] Picozza P., Galper A.M., \& Castellini, G. et al. 2007, APh, 27, 296

[14] Adriani, O., Barbarino, G. C., \& Bazilevskaya, G. A. et al., 2015, submitted to ApJ

[15] Gorski, K. M., Hivon, E., \& Banday, A. J. et al. 2005, ApJ, 622, 759

[16] Li, T.P., \& Ma, Y.Q. 1983, ApJ, 272, 317

[17] Bruno, A., Adriani, O., \& Barbarino, G. C. et al. 2014, arXiv:1412.1765

[18] Finlay, C. C., Maus, S., \& Begganet, C. D. et al. 2010, GeoJI, 183, 1216

[19] Tsyganenko, N. A., \& Sitnov, M. I. 2005, JGR, 110, A03208 